\title{
NOTE ON HADAMARD'S DETERMINANT THEOREM
}

\section{JOHN WILLIAMSON}

Introduction. We shall call a square matrix $A$ of order $n$ an Hadamard matrix or for brevity an $H$-matrix, if each element of $A$ has the value \pm 1 and if the determinant of $A$ has the maximum possible value $n^{n / 2}$. It is known that such a matrix $A$ is an $H$-matrix [1] ${ }^{1}$ if, and only if, $A A^{\prime}=n E_{n}$ where $A^{\prime}$ is the transpose of $A$ and $E_{n}$ is the unit matrix of order $n$. It is also known that, if an $H$-matrix of order $n>1$ exists, $n$ must have the value 2 or be divisible by 4 . The existence of an $H$-matrix of order $n$ has been proved [2,3] only for the following values of $n>1$ : (a) $n=2$, (b) $n=p^{h}+1 \equiv 0 \bmod 4, p$ a prime, (c) $n$ $=m\left(p^{h}+1\right)$ where $m \geqq 2$ is the order of an $H$-matrix and $p$ is a prime, (d) $n=q(q-1)$ where $q$ is a product of factors of types (a) and (b), (e) $n=172$ and for $n$ a product of any number of factors of types (a), (b), (c), (d) and (e).

In this note we shall show that an $H$-matrix of order $n$ also exists when (f) $n=q(q+3)$ where $q$ and $q+4$ are both products of factors of types (a) and (b), (g) $n=n_{1} n_{2}\left(p^{h}+1\right) p^{h}$, where $n_{1}>1$ and $n_{2}>1$ are orders of $H$-matrices and $p$ is an odd prime, and (h) $n=n_{1} n_{2} m(m+3)$ where $n_{1}>1$ and $n_{2}>1$ are orders of $H$-matrices and $m$ and $m+4$ are both of the form $p^{h}+1, p$ an odd prime.

It is interesting to note the presence of the factors $n_{1}$ and $n_{2}$ in the types $(\mathrm{g})$ and $(\mathrm{h})$ and their absence in the types (d) and (f). Thus, if $p$ is a prime and $p^{h}+1 \equiv 0 \bmod 4$, an $H$-matrix of order $p^{h}\left(p^{h}+1\right)$ exists but, if $p^{h}+1 \equiv 2 \bmod 4$, we can only be sure of the existence of an $H$-matrix of order $n_{1} n_{2} p^{h}\left(p^{h}+1\right)$ where $n_{1}>1$ and $n_{2}>1$ are orders of $H$-matrices. This is analogous to the simpler result that, if $p^{h}+1 \equiv 0$ $\bmod 4$ an $H$-matrix of order $p^{h}+1$ exists but, if $p^{h}+1 \equiv 2 \bmod 4$, we can only be sure of the existence of an $H$-matrix of order $n\left(p^{h}+1\right)$ where $n>1$ is the order of an $H$-matrix.

We shall denote the direct product of two matrices $A$ and $B$ by $A \cdot B$ and the unit matrix of order $n$ by $E_{n}$.

Theorems on the existence of $H$-matrices. If a symmetric $H$-matrix of order $m>1$ exists, there exists an $H$-matrix $H$ of order $m$ with the form

$$
H=\left(\begin{array}{ll}
1 & e \\
e^{\prime} & D
\end{array}\right),
$$

Received by the editors December 6, 1946.

1 Numbers in brackets refer to the references cited at the end of the paper. 
where $e$ is the row vector $(1,1, \cdots, 1)$ of dimension $m-1$ and $e^{\prime}$ the column vector which is the transpose of $e$. Since $H$ is a symmetric $H$-matrix

$$
H^{2}=H H^{\prime}=m E_{m}
$$

and accordingly

$$
\left(\begin{array}{cc}
m & e+e D \\
e^{\prime}+D e^{\prime} & e^{\prime} e+D^{2}
\end{array}\right)=\left(\begin{array}{cc}
m & 0 \\
0 & m E_{m-1}
\end{array}\right) .
$$

Therefore

$$
e D=-e, \quad D e^{\prime}=-e^{\prime}
$$

and

$$
D^{2}=m E_{m-1}-R,
$$

where

$$
R=e^{\prime} e
$$

and $R$ is the square matrix of order $m-1$ each element of which has the value 1 . It follows easily that

$$
R^{2}=(m-1) R
$$

and by (1) and (3) that

$$
R D=-R=D R .
$$

If $F=2 E_{m-1}-R, F$ is a symmetric matrix each element of which has the value \pm 1 . Further

$$
F D=D F
$$

by (5) and

$$
F^{2}=4 E_{m-1}+(m-5) R
$$

by (4). If $n$ is a product of factors of types (a) and (b) there exists $\left[3\right.$, p. 67] an $H$-matrix of order $n$ with the form $E_{n}+S$ where $S$ is skew-symmetric so that

$$
S^{2}=-(n-1) E_{n} .
$$

If

$$
W=F \cdot E_{n}+D \cdot S,
$$

each element of $W$ has the value \pm 1 and 


$$
\begin{array}{rlr}
W W^{\prime} & =\left(F \cdot E_{n}+D \cdot S\right)\left(F \cdot E_{n}-D \cdot S\right) \\
& =F^{2} \cdot E_{n}-D^{2} \cdot S^{2} & (\text { by }(6)) \\
& =\left[4 E_{m-1}+(m-5) R\right] \cdot E_{n}+\left(m E_{m-1}-R\right) \cdot(n-1) E_{n} \\
& =\left[(4+m n-m) E_{m-1}\right] \cdot E_{n}+(m-n-4) R \cdot E_{n} .
\end{array}
$$

Therefore, if $n=m-4$,

$$
W W^{\prime}=(m-1)(m-4) E_{m-1} \cdot E_{m-4}=n(n+3) E_{n} \cdot E_{n+3}
$$

and $W$ is an $H$-matrix.

Since a symmetric $H$-matrix of order 2 exists and a symmetric $H$-matrix of order $p^{h}+1 \equiv 0 \bmod 4$, where $p$ is a prime, exists [3, p. 67], there exists a symmetric $H$-matrix of order $n$ where $n$ is a product of factors of types (a) and (b). We have therefore proved the theorem:

THEOREM 1. If $n$ and $n+4$ are both products of factors of types (a) and (b) there exists an $H$-matrix of order $n(n+3)$.

As a particular case of this theorem we have the corollary:

CoROLlaRy 1. If $n-1$ and $n+3$ are both powers of primes and are congruent to 3 modulo 4 , there exists an $H$-matrix of order $n(n+3)$.

If $m=p^{h}+1 \equiv 2 \bmod 4$, where $p$ is a prime, there exists $[3, p .66]$ a symmetric matrix $T$ of order $m$, each diagonal element of which has the value 0 and each other element the value \pm 1 and such that

$$
T=\left(\begin{array}{rr}
0 & e \\
e^{\prime} & U
\end{array}\right)
$$

and

$$
T^{2}=(m-1) E_{m}
$$

It follows therefore that

$$
U U^{\prime}=U^{2}=(m-1) E_{m-1}-R,
$$

where $R$ is defined by (3). Let $A_{1}$ and $B_{1}$ be two $H$-matrices of order $n_{1}$ such that $[3$, p. 66]

$$
A_{1} B_{1}^{\prime}=-B_{1} A_{1}^{\prime}
$$

and let $\mathrm{K}=A_{1} \cdot E_{m-1}+B_{1} \cdot U$. Then each element of $\mathrm{K}$ has the value \pm 1 and 


$$
\begin{aligned}
\mathrm{KK}^{\prime} & =A_{1} A_{1}^{\prime} \cdot E_{m-1}+B_{1} B_{1}^{\prime} \cdot U^{2} \\
& =n_{1} E_{n_{1}} \cdot\left(m E_{m-1}-R\right)
\end{aligned}
$$

Since $e U=0=U e^{\prime}$,

$$
R U=U R=0
$$

Hence, if $\Gamma=A_{1} \cdot R$,

$$
\Gamma \Gamma^{\prime}=n_{1} E_{n_{1}} \cdot(m-1) R
$$

and

$$
\Gamma \mathrm{K}^{\prime}=A_{1} A_{\mathrm{i}}^{\prime} \cdot R=\mathrm{K}^{\prime}
$$

Finally, if $A_{2}$ and $B_{2}$ are two $H$-matrices of order $n_{2}$ satisfying

$$
A_{2} B_{2}^{\prime}=-B_{2} A_{2}^{\prime} \text {, }
$$

and

$$
\begin{array}{rlr}
W & =A_{2} \cdot \Gamma \cdot E_{m}+B_{2} \cdot \mathrm{K} \cdot T, \\
W W^{\prime} & =A_{2} A_{2}^{\prime} \cdot \Gamma \Gamma^{\prime} \cdot E_{m}+B_{2} B_{2}^{\prime} \cdot \mathrm{KK}^{\prime} \cdot T^{2} & (\text { by }(15) \text { and (16)) } \\
& =n_{2} E_{n_{2}} \cdot n_{1} E_{n_{1}} \cdot\left[(m-1) R+\left(m E_{m-1}-R\right)(m-1)\right] \cdot E_{m} \\
& =r E_{r} & (\text { by }(9),(12) \text { and (14)) } \\
& & \left(r=n_{1} n_{2} m(m-1)\right) .
\end{array}
$$

Therefore $W$ is an $H$-matrix and we have proved the theorem:

THEOREM 2. If $H$-matrices of orders $n_{1}$ and $n_{2}$ exist, $n_{1}>1, n_{2}>1$, and $p$ is a prime such that $p^{h}+1 \equiv 2 \bmod 4$, there exists an $H$-matrix of order $n_{1} n_{2} p^{h}\left(p^{h}+1\right)$.

Since, if $p$ is a prime such that $p^{h}+1 \equiv 0 \bmod 4$, there exists an $H$-matrix of order $p^{h}\left(p^{h}+1\right)$, we have the corollary:

CoROLlaRy 1. If $H$-matrices exist of orders $n_{1}>1$ and $n_{2}>1$, there exists an $H$-matrix of order $n_{1} n_{2}\left(p^{h}+1\right) p^{h}$ where $p$ is an odd prime.

Since an $H$-matrix of order 2 exists we have the corollary:

CoRollary 2. If $p$ is an odd prime an H-matrix exists of order $4 p^{h}\left(p^{h}+1\right)$.

In the proof of the final theorem we require the following lemma:

LEMMA 1. If there exists an $H$-matrix $A$ of order $n>1$, there exist two $H$-matrices $B$ and $C$ of order $n$ such that $A B^{\prime}=-B A^{\prime}, A C^{\prime}=C A^{\prime}$, $B C^{\prime}=C B^{\prime}$. 
In fact the matrices $B=X A$ and $C=Y A$, where $X$ is the diagonal block matrix

$$
\left[\left(\begin{array}{rr}
0 & 1 \\
-1 & 0
\end{array}\right),\left(\begin{array}{rr}
0 & 1 \\
-1 & 0
\end{array}\right), \cdots,\left(\begin{array}{rr}
0 & 1 \\
-1 & 0
\end{array}\right)\right]
$$

and $Y$ is the diagonal block matrix

$$
\left[\left(\begin{array}{ll}
0 & 1 \\
1 & 0
\end{array}\right),\left(\begin{array}{ll}
0 & 1 \\
1 & 0
\end{array}\right), \cdots,\left(\begin{array}{ll}
0 & 1 \\
1 & 0
\end{array}\right)\right],
$$

satisfy the conditions of the lemma. For $B B^{\prime}=C C^{\prime}=n E_{n}, A B^{\prime}=n X^{\prime}$ $=-n X=-B A^{\prime}, \quad C A^{\prime}=n Y^{\prime}=n Y=C A^{\prime} \quad$ and $\quad B C^{\prime}=n X Y^{\prime}=n Y X^{\prime}$ $=C B^{\prime}$.

Let $\mathrm{M}=C_{1} \cdot\left(2 E_{m-1}-R\right)$ and $\mathrm{N}=A_{1} \cdot E_{m-1}+B_{1} \cdot U$, where $R$ is defined by (14), $U$ by (10) and $A_{1}, B_{1}$ and $C_{1}$ are matrices of order $n_{1}$ with the properties of Lemma 1 . Then each element of the matrices $\mathrm{M}$ and $\mathrm{N}$ has the value \pm 1 . Further

$$
\begin{aligned}
& \mathrm{MM}^{\prime}=n_{1} E_{n_{1}} \cdot\left(4 E_{m-1}-4 R+R^{2}\right) \\
& =n_{1} E_{n_{1}} \cdot\left[4 E_{m-1}+(m-5) R\right] \\
& \mathrm{NN}^{\prime}=n_{1} E_{n_{1}} \cdot\left(E_{m-1}+U^{2}\right)=n_{1} E_{n_{1}} \cdot\left(m E_{m-1}-R\right) \quad(\text { by }(10))
\end{aligned}
$$

and

$$
\begin{aligned}
\mathrm{MN}^{\prime} & =C_{1} A_{1}^{\prime} \cdot\left(2 E_{m-1}-R\right)+C_{1} B_{1}^{\prime} \cdot\left(2 E_{m-1}-R\right) U \\
& =C_{1} A_{1}^{\prime} \cdot\left(2 E_{m-1}-R\right)+C_{1} B_{1}^{\prime} \cdot 2 U
\end{aligned}
$$

Therefore by Lemma 1

$$
\mathrm{MN}^{\prime}=\mathrm{NM}^{\prime} \text {. }
$$

Let $A_{2}$ and $B_{2}$ be two $H$-matrices of order $n_{2}>1$ satisfying (16) and let $n=p^{h}+1 \equiv 2 \bmod 4$ where $p$ is a prime. Then there exists a matrix $G$ of order $n$ and of the same form as $T$ in (9) and satisfying

$$
G^{2}=(n-1) E_{n} \text {. }
$$

If finally $W=A_{2} \cdot \mathbf{M} \cdot E_{n}+B_{2} \cdot \mathrm{N} \cdot G$, each element of $W$ has the value \pm 1 and

$$
\begin{array}{rlr}
W W^{\prime}= & n_{2} E_{n_{2}} \cdot\left(\mathrm{MM}^{\prime} \cdot E_{n}+\mathrm{NN}^{\prime} \cdot G^{2}\right) & (\text { by }(16) \text { and (19)) } \\
= & n_{1} n_{2} E_{n_{1} n_{2}} \cdot\left[4 E_{m-1}+(m-5) R\right] \cdot E_{n} & \\
& +\left(m E_{m-1}-R\right) \cdot(n-1) E_{n} & (\text { by }(17),(18) \text { and (20)) } \\
= & n_{1} n_{2} E_{n_{1} n_{2}} \cdot\left[(4+m n-m) E_{m-1}+(m-4-n) R\right] \cdot E_{n} .
\end{array}
$$


Hence, if $m=n+4$ and $r=n_{1} n_{2} n(n+3)=n_{1} n_{2}(m-1)(m-4)$,

$$
W W^{\prime}=r E_{r}
$$

and $W$ is an $H$-matrix. We have therefore proved the theorem:

THEOREM 3. If $n$ and $n+4$ are both of the form $p^{h}+1 \equiv 2 \bmod 4$ where $p$ is a prime and if $H$-matrices of orders $n_{1}>1$ and $n_{2}>1$ both exist, there exists an H-matrix of order $n_{1} n_{2} n(n+3)$.

As a consequence of Theorem 1 we have the corollary:

CoROLLARY 1. If $n$ and $n+4$ are both of the form $p^{h}+1$ where $p$ is an odd prime and if $H$-matrices of orders $n_{1}>1$ and $n_{2}>1$ both exist, there exists an $H$-matrix of order $n_{1} n_{2} n(n+3)$.

Since an $H$-matrix of order 2 exists we also have the corollary:

CoRollaRy 2. If $n$ and $n+4$ are both of the form $p^{n}+1$, where $p$ is an odd prime, there exists an H-matrix of order $4 n(n+3)$.

Particular examples. That the above theorems do actually increase the values of $n$ as orders of $H$-matrices which are known to exist is shown by the following examples.

By Theorem 1 an $H$-matrix of order (56)(59) exists. For $56=2\left(3^{3}+1\right)$ and 59 is prime. Further no one of (56)(59), (28)(59), (14)(59), 4(59) or $2(59)$ is of the form $p^{h}+1$. Therefore (56)(59) is not a product of factors of types (a), (b) or (c). By Theorem 2 an $H$-matrix of order 4(73)(74) exists and by Theorem 3 an $H$-matrix of order 4(230)(233) exists. Neither of the numbers $4(73)(74)$ nor $4(230)(233)$ is a product of factors of types (a), (b), (c) and (d).

\section{REFERENCES}

1. Jacques Hadamard, Résolution d'une question relative aux déterminants, Bull. Sci. Math. (2) vol. 17 (1893) pp. 240-246.

2. R. E. A. C. Paley, On orthogonal matrices, Journal of Mathematics and Physics, Massachusetts Institute of Technology, vol. 12 (1933) pp. 311-320.

3. John Williamson, Hadamard's determinant theorem and the sum of four squares, Duke Math. J. vol. 11 (1944) pp. 65-81.

Queens College 